\title{
TRIBOLOGICAL BEHAVIOUR OF POLYMER BEARINGS UNDER DRY AND WATER LUBRICATION
}

\author{
TD. Nguyen ${ }^{1}$; J. Sukumaran ${ }^{1}$; J. De Pauw ${ }^{1}$; P. De Baets ${ }^{1}$ \\ ${ }^{1}$ Ghent University, Laboratory Soete, Belgium
}

\begin{abstract}
This study attempts to evaluate the performance of polymer journal bearings (PET and UHMWPE) sliding against Inconel stainless steel in both dry and lubricated condition. Four types of 'lubricant' are included: distilled water; demineralized water; tap water and river water. The tests were carried out in a so called "Stribeck" configuration with a projected pressure of about $0.3 \mathrm{MPa}$ at room temperature and sliding speed ranging from 0 to $1.07 \mathrm{~m} / \mathrm{s}$ respectively. The study indicates that polymer bearings with four types of water lubricant give better tribological behaviour compared to those in dry sliding contact. Besides, with water lubrication, UHMWPE shows low friction at starting, but it does not go down much over the course of the tests. Meanwhile, although PET indicates quite higher friction at the beginning, it then shows a clearly decreasing trend.
\end{abstract}

Keywords: water lubrication, polymer bearings, friction.

\section{INTRODUCTION}

Journal bearings are one of the most important machine elements and are designed to operate in fully hydrodynamic regime where a thick lubricating film separates the sliding and stationary surfaces. Traditionally, journal bearings are made from metal and lubricated by oil or grease. Polymers and polymers-based composites have proved good tribological performances thanks to their corrosion resistant properties and the ability of embedding foreign matters such as wear particles [1]. In applications where bearings operate in aqueous medium such as hydro power plants, pumps, marine ship propellers....polymer bearings lubricated by (sea) water possibly provide a good solution. The commonly used polymer bearings in such applications are UHMWPE, PA6, POM,...

Ultra-High Molecular Weight Polyethylene (UHMWPE) is used as bearing material characteristics such as wear resistance, low friction, high impact strength, ultra-low water absorption and excellent chemical stability in various corrosive environments, making it potentially suitable for tribological application in aqueous environment [2]. Polyethylene terephthalate (PET) is an engineering thermoplastic which is mostly used as commodity thermoplastic due to its low cost. Its low cost, large availability and wear resistance in aqueous environment [3] makes PET a potential material for application in water lubricated bearings.

Tribological behaviour of polymers including UHMWPE and PET under water lubrication depend on many factors such as properties of lubricants, mating materials and operating conditions [4]. The viscosity of water is more than 100 times lower than that of oil. This means that in most cases with water lubrication, bearings operate during a considerable part of their service life under boundary or mixed regime [5]. As a consequence, good understanding of bearings under these regimes is important.

Limited research has been done on polymer bearings lubricated by water. This study therefore aims at comparing of the effect of four types of water lubricants (distilled, demineralized, tap and river) on tribological properties of PET and UHMWPE bearings.

\section{EXPERIMENTAL DETAILS}

\subsection{Sample materials}

PET and UHMWPE are bought from a commercial market in form of circular bar. The bar stock is then machined to the required dimension. The mating material (shaft) is Inconel which is machined and 
then polished by 400 grit size abrasive paper to obtain a $R_{a}$ of $0.2 \mu \mathrm{m}$. The diameter of the shaft and inner diameter of bearing are $30(\mathrm{~mm})$. More details are listed in table 1.

Table 1. Parameters of bearings

\begin{tabular}{|l|c|c|c|}
\hline Sample & Clearance $\mathbf{( m m})$ & Width $\mathbf{~} \mathbf{m m})$ & Inner diameter $\mathbf{( m m})$ \\
\hline PET & 0.1 & 15 & 30 \\
\hline UHMWPE & 0.1 & 15 & 30 \\
\hline
\end{tabular}

\subsection{Lubricants}

In this test, four types of lubricant were used, namely distilled water (from the comercial market), demineralized water, tap water and river water (in Ghent). Water was supplied continuosly in such a way that there was always water in the contact surface between bearing and shaft.

\subsection{Test rig}

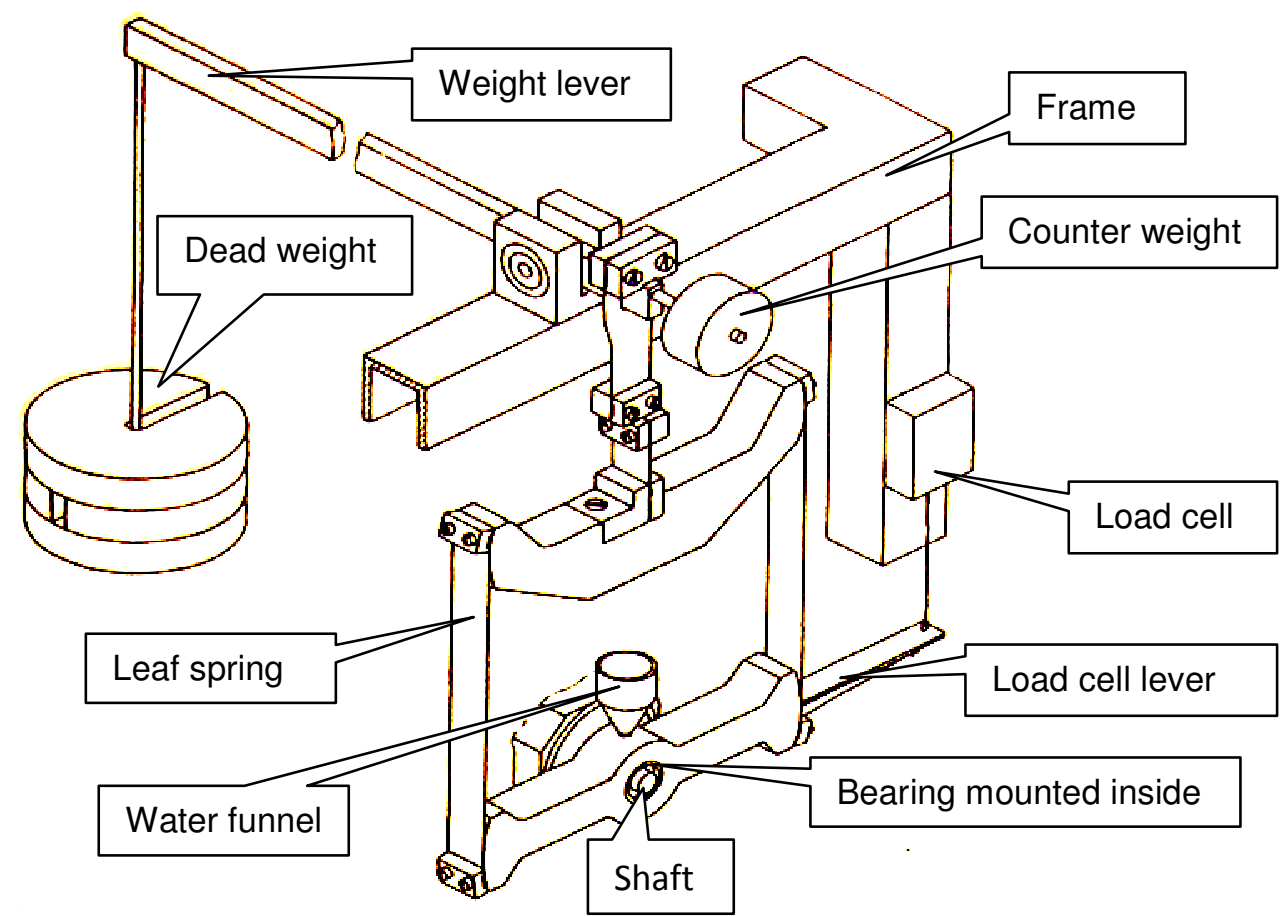

Figure 1. Schematic diagram of Stribeck test rig with its main components

The tests were carried out on a newly developed Stribeck test rig in Labo Soete. Figure 1 shows a schematic diagram of the Stribeck test rig where radial load $\left(F_{R}\right)$ is applied to the bearing by using suspended dead weight and lever mechanism. The friction force or the circumferential force $\left(F_{C}\right)$ on the surface of bearing is calculated based on the force $\left(F_{L}\right)$ acquired by the load cell (figure 2). The coefficient of friction $(\mu)$ can be calculated using the equation (1),(2) \& (3). A thermal couple is mounted in the shaft records the temperature rise in the shaft during sliding. 


$$
\begin{gathered}
F_{R}=\frac{500 \mathrm{~mm}}{50 \mathrm{~mm}} * F_{D} \\
F_{C}=\frac{a}{r} * F_{L} \\
\mu=\frac{\mathrm{F}_{\mathrm{C}}}{\mathrm{F}_{\mathrm{R}}}
\end{gathered}
$$

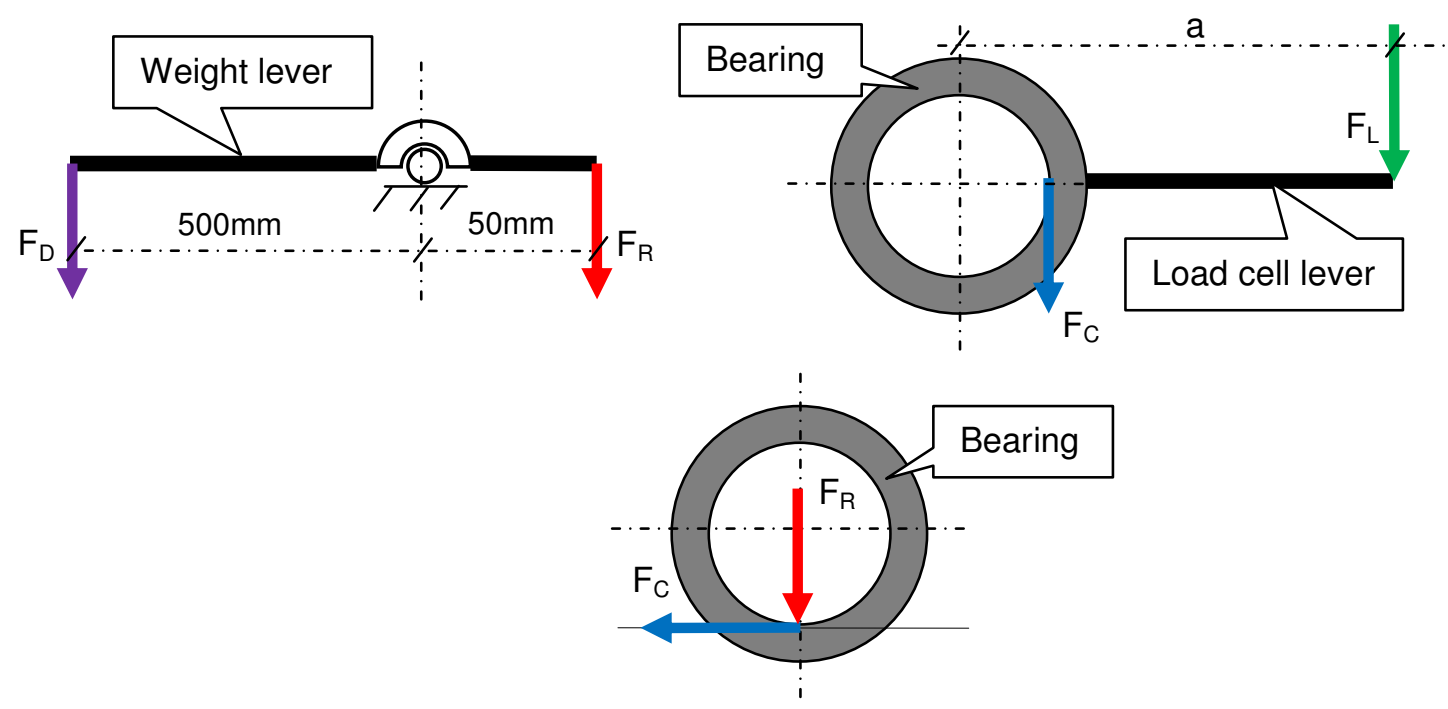

Figure 2. Free body analyses

Labview ${ }^{\circledR}$ program is used to controll the test rig using a $D A Q$. All measurements were recoded online using NI USB 6009 data acquisition. Running in was chosen to have conformity between the bearing and the mating surface. The preliminary test showed that a running in is sufficient in 4 hours by having steady friction coefficient.

\subsection{Methodology}

The following test sequences were used in our current investigation:

- Running-in: 4 hours

- Break-away friction and boundary friction: 20 minutes at low speed $(0.055 \mathrm{~m} / \mathrm{s})$

- Ramping characteristics : 40 minutes with increasing linear speed (0-1.07 m/s) or (0-680 rmp)

Dry sliding contact was just done to understand the break-away and boundary friction. This is because of the fact that at high speed, the temperature on dry sliding contact will increase and thus melt polymer bearings.

\subsection{Break-away friction and boundary friction}

Break- away friction is the maximum static friction just before sliding. To get information on the breakaway and boundary friction, the tests were carried out at extremely slow sliding speeds to avoid the possibility of hydrodynamic effects. One test consisted of 100 cycles of 12 seconds each. Figure 3 
gives an example of the raw data about the break-away and boundary friction of the first two cycles when testing UHMWPE in dry contact. In each cycle, the maximum friction and boundary friction were recoded. The break- away friction is the peak value at the beginning of the cycle. An average of these peak values and boundary values was made to determine the break-away and boundary friction for the materials under different testing conditions.

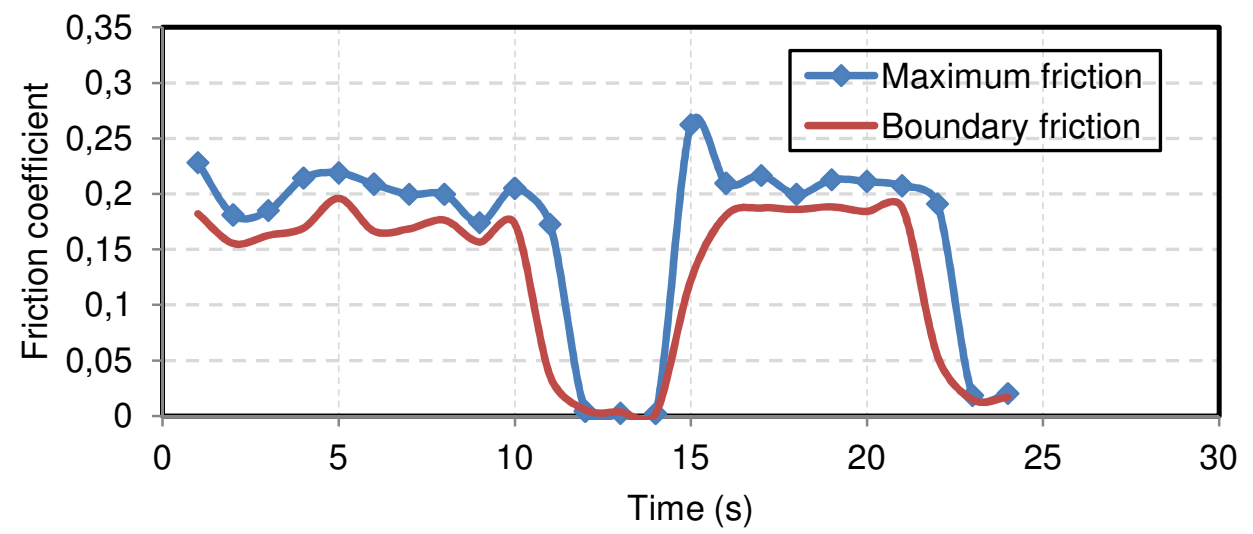

Figure 3. Friction curve over $24 \mathrm{~s}$ (first 2 cycles) from testing of UHMWPE in dry contact

\section{RESULTS AND DISCUSSION}

Based on results from the literature [ 6-9], it is expected that the lubricated condition at a speed of 0.1 $(\mathrm{m} / \mathrm{s})$ ensures boundary lubrication and that a sliding speed of $(0.01 \mathrm{~m} / \mathrm{s})$ ensures perfect boundary lubrication. In our current tests, the break away and the boundary friction were determined at speed of about $0.055 \mathrm{~m} / \mathrm{s}$ which is corresponding to about $35 \mathrm{rpm}$ ( the lowest rpm at which the motor can rotate.

As observed from figure $4 \& 5$, the coefficient of friction in dry contact is higher than that in all lubricated conditions. This difference in coefficient of friction is more obvious in case of boundary conditions. This confirms that water lubrication can result friction reduction for polymer bearings.

\subsection{Break-away friction (BAF)}

Investigating on the start-up in sliding bearing bearings, it was found that journal bearings rapidly become hydrodynamic following an initial moment of break-away friction [10].Therefore, a reduction in break-away friction, in practical situations often related to start and stop conditions of mechanical equipment, is expected to avoid bearing failure.

Figure 4 shows the break-away coefficient of friction two polymer bearings (PET and UHMWPE) sliding against Inconel stainless steel under dry contact and under distilled water, demineralized water, tap water and river water respectively. It can be seen that BAF of UHMWPE bearing is approximately two times less than that of PET bearing in corresponding contacts.

For PET bearing, demineralized water provides less BAF than others while river water shows highest friction among the lubricants. Distilled water and tap water have almost the same BAF of order of 0.65 .

A small difference in BAF for all water types is seen while using UHMWPE bearing. In contrary to PET bearings, river water provides lowest BAF and the difference in BAF is not remarkable between dry sliding contact and lubricated cases. 


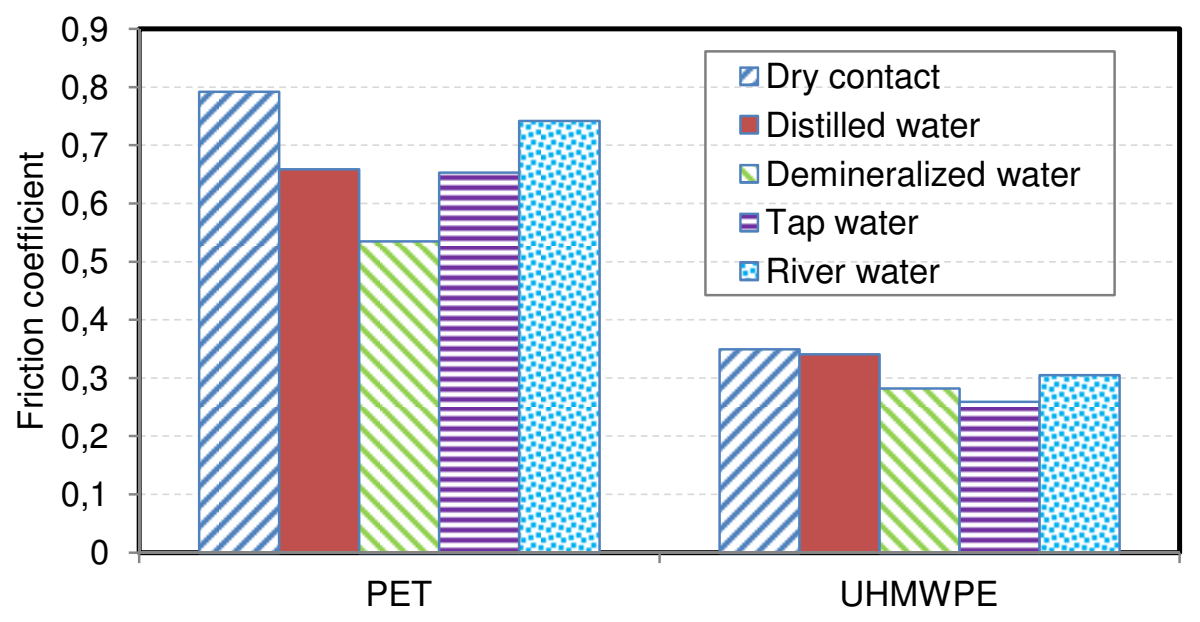

Figure 4. Break- away friction coefficient of two polymer bearings sliding against Inconel steel under dry contact and under distilled water, demineralized water, tap water and river water

\subsection{Boundary friction $(B F)$}

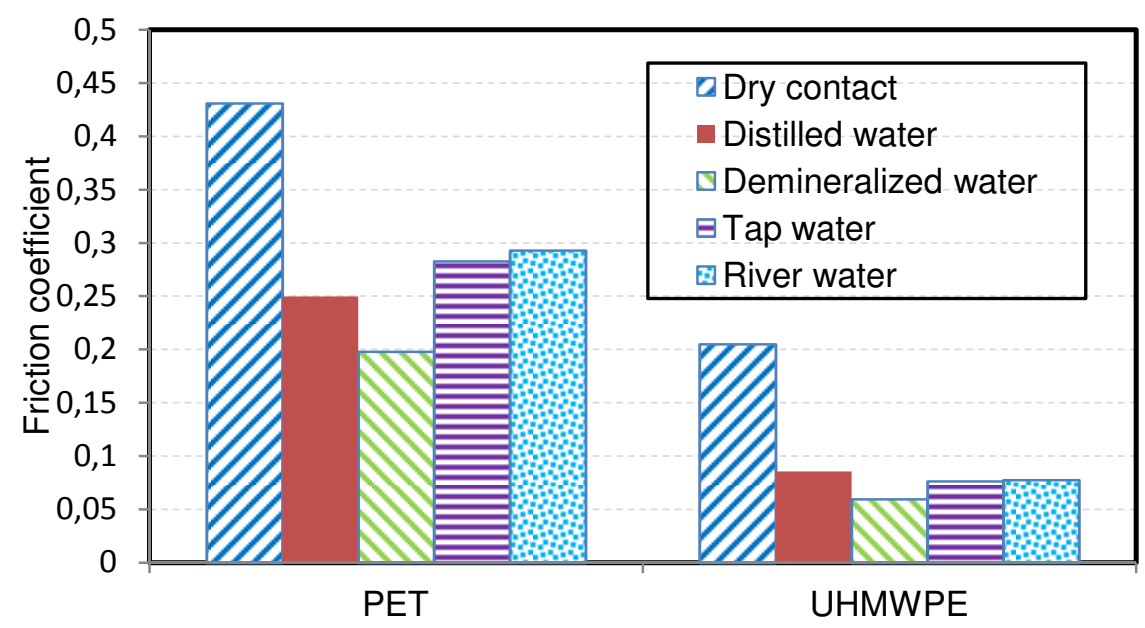

Figure 5. Boundary friction coefficient of two polymer bearings sliding against Inconel steel with dry contact and under distilled water, demineralized water, tap water and river water

Within a cycle of a test, the boundary friction has more values recorded than break-away friction. Therefore, the boundary coefficient of friction of two polymer bearings sliding against Inconel steel with dry contact and under distilled water, demineralized water, tap water and river water figure4 will reflect lubricating effects more precisely than the case of break-away friction. Based on figure 4, one can see that under dry sliding contact, the boundary friction is almost twice higher than that under lubricating situations for both bearing materials. The results for UHMWPE are more convincing than the PET results. This is because UHMWPE has poor thermal properties; it is liable to softening under dry sliding contact where there is a presence of a large amount of friction-induced heat. As a result, UHMWPE usually shows high coefficient of friction. Meanwhile, under aqueous medium lubrication, friction-induced heat can be effectively taken away by the medium [1] resulting in low friction.

For PET bearing, the coefficient of friction under demineralized water lubrication is lowest $(0,2)$, even lower than half that under dry contact. The friction under distilled water is a little bit higher about 0.25 and followed by both tap water and river water which are nearly equal to each other.

In case of UHMWPE bearing, friction does not depend much on water type. Demineralized water gives smallest friction again while other three are slightly larger and stay almost the same value. 


\subsection{Evolution of coefficient of friction against speed}

Figure 6 represents the curve of coefficient of friction with changing the speed of the shaft. When the speed increases from low rpm to maximum rpm, the lubrication regime moves from boundary to mixed lubrication. At higher speed, the increasing of water film or decreasing of the extent of direct contact between the rubbing surfaces results into lower friction. However, the bearing is still not running in hydrodynamic regime because the friction value is greater than 0.02 for both materials. It is obvious that when the shaft speed increases, the friction decreases faster with PET than with UHMWPE. And also, at maximum speed, the coefficients of friction under four types of water lubrication for PET bearing are quite close to each other while they are rather different for UHMWPE bearing where demineralized water provides smallest friction of about 0.045 compared to the largest one by tap water (0.075).

For PET bearing, demineralized water and river water provide lower friction than that with distilled water and tap water, but all four water types show quite similar friction at high speed (over $635 \mathrm{rpm}$ ).

The variation of friction of UHMWPE bearing with the increasing speed is not very significant. At maximum speed, the friction with four waters is not exactly the same as in case of PET bearing. Demineralized water still gives lowest friction while tap water provides the highest one. Distilled water and tap water show quite similar friction at maximum speed although their values at low speed are different.

The graph shapes are of greater importance than the values themselves because they proves that water film is formed when sliding speed increases.
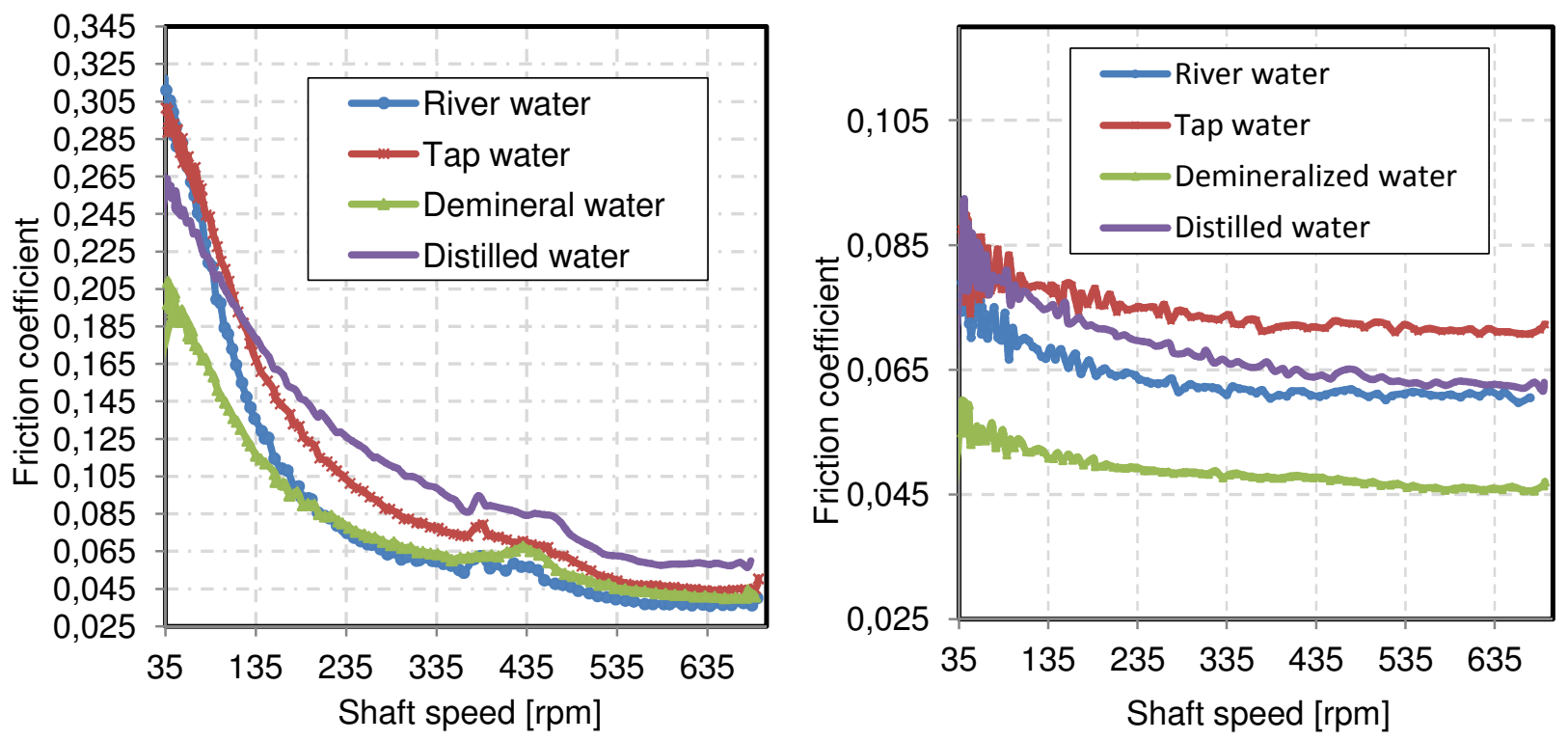

Figure 6. Friction coefficient vs. shaft speed (rpm) of PET (left) and UHMWPE (right)

\section{NOMENCLATURE}

$\mathrm{F}_{\mathrm{R}} \quad$ Radial load 


$\begin{array}{lll}F_{C} & \text { Circumferential force or friction force } & N \\ F_{D} & \text { Weight of the dead weight } & N \\ F_{L} & \text { Forced captured by the load cell } & N \\ \mu & \text { friction coefficient } & \\ r & \text { inner radius of bearing } & m \\ a & \text { Distance from bearing center to load cell } & m\end{array}$

5. CONCLUSIONS

- The friction for PET bearing is significantly larger than that for UHMWPE in all the cases.

- The friction under lubricated conditions is low than under dry sliding contact, especially in the case of boundary lubrication.

- Among the four types of water, demineralized water provides the purities friction characteristics for bearing applications.

- Hydrodynamic lubrication is easier to be achieved with PET than with UHMWPE

- At high speed (maximum), the friction observed on PET is very similar for four lubricating waters while a significant difference is observed with UHMWPE.

\section{REFERENCES}

[1] Jianzhang Wang . Fengyuan Yan . Qunji Xue, Friction and Wear Behavior of Ultra-high Molecular Weight Polyethylene Sliding Against GCr15 Steel and Electroless Ni-P Alloy Coating Under Lubrication of Sea Water. Tribological Letter, 35, 85-95, 2009.

[2] Xiong, D.S.,Gao, Z., Jin, Z.M, Friction and Wear Properties of UHMWPE Against lon Implanted Titanium Alloy. Surface \& Coatings Technology 201,6847-6850,2007

[3] C.G. Clarke, C. Allen, The Water Lubricated, Sliding Wear Behavior of Polymeric Materials Against Steel, Tribology International, Vol. 24, Issue 2, 109-118, 1991

[4] Jianzhang Wang . Fengyuan Yan . XUE Qunji, Tribological Behaviors of Some Polymeric Materials In Sea Water. Chinese Science Bulletin,2009

[5] B.M. Ginzburg, D.G. Tochil'nokov, V.E. Bakhareva, A.V. Anisimov, and O.F. Kireenko, Polymeric Materials for Water-lubricated Plain Bearings, Russian journal of applied chemistry, Vol.79, No. 5, 2006

[6] K. Tanaka, Friction and Wear of Semicrystalline Sliding against Steel Under Water Lubrication, J. Lubrication Technology, 102 , 526, 1980

[7] S. W. Lasoski, Jr., and W. H. Cobbs, Moisture Permeability of Polymers, I, Role of crystallinity and orientation, J. Polym. Sci, 36, 21, 1959

[8] Y. Yamada and K. Tanaka, Effect of The Degree of Crystallinity on Friction and Wear of Poly(ethylene terephthalate), In L. H. Lee (ed.), Polymer Wear and Its Control, in Am. Chem. Sot. Symp. Ser, 287 Chapter 24, 1985

[9] Y. Yamada and K. Tanaka, Friction and Wear Behaviour of PTFE-Based Composites Under Water Lubrication, Zyunkatsu, 29, 209, 1984

[10] BouyerJ, Fillon M, Valle V, Stick-Slip Phenomenom Induced by Friction in a Plain Journal Bearing During Start-up. In World tribology congress, Kyoto, Japan, 101, 2009 